\title{
ON A DIFFERENTIAL INEQUALITY
}

WOODROW W. BLEDSOE, MICHAEL J. NORRIS, AND GENE F. ROSE

1. Introduction. Throughout this paper capital Latin letters will denote matrices. In particular, $F$ and $G$ will denote $n \times 1$ matrices, that is, column vectors. The letter $Y$ will be used for the $n \times 1$ matrix of the coordinates of a variable in $n$ dimensions. The special $n \times 1$ matrices having 0 everywhere or 1 everywhere will be denoted by $\theta$ and $\alpha$, respectively.

The components of a matrix $M$ will be denoted by $m_{i j}$, or in the case of a column vector simply by $m_{i}$. The positive and negative parts of a matrix or scalar will be indicated by attaching the subscripts 1 and 2 respectively. While these two notations are not compatible, the meaning will always be clear from the context.

The upper right derivative operator is denoted by $D^{+}$.

We are mainly concerned in this paper with determining fairly general conditions which together with $F(a) \geqq \theta$ and $D^{+} F \geqq M F$ on the interval $a \leqq x \leqq b$ insure that $F \geqq \theta$ on this interval. Results along this line are obtained, and examples are given that indicate that these results are about as general as possible. Finally some immediate consequences of these results are derived in regard to somewhat more general inequalities.

2. The linear case. In this and succeeding sections it is always understood to be part of the hypotheses of the theorems and lemmas that the number of rows and the number of the columns of the matrices involved are such as to permit the indicated matrix multiplications and additions. Usually the independent variable is $x$, but in some cases $(Y, x)$. Differentiations are with respect to $x$. Functions are assumed to be finite-valued when given, but their derivatives need not be finite-valued.

Lemma 1. On the interval $[a, b]$ let:

(1) $F$ be continuous, $D^{+} F$ never be $-\infty$ for any component, and $F(a) \geqq \theta$;

(2) $M$ be non-negative almost everywhere and essentially bounded above (that is, there is a c such that, for all $i$ and $j, m_{i j} \leqq c$ almost everywhere);

(3) $D^{+} F \geqq M F$ almost everywhere. Then $F \geqq \theta$ on $[a, b]$.

Presented to the Society, February 27, 1954; received by the editors March 12, 1954. 
Proof. As guaranteed by (2) let $c$ be non-negative and an upper bound almost everywhere for the components of $M \alpha$.

Since $F$ is continuous on $[a, b]$, we have the components of $F$ bounded there. By (2) and (3) there is a negative number $r$ such that $D^{+} F \geqq r \alpha$ almost everywhere.

We now show by mathematical induction that for every non-negative integer $n, D^{+} F \geqq\left(r c^{n}(x-a)^{n} / n !\right) \alpha$ almost everywhere. We already have the result for $n=0$.

Suppose then that for the non-negative integer $k, D^{+} F$ $\geqq\left(r c^{k}(x-a)^{k} / k !\right) \alpha$ almost everywhere. We have

$$
D^{+}\left(F-\frac{r c^{k}(x-a)^{k+1}}{(k+1) !} \alpha\right)=D^{+} F-\frac{r c^{k}(x-a)^{k}}{k !} \alpha \geqq \theta
$$

almost everywhere,

$$
\begin{gathered}
D^{+}\left(F-\frac{r c^{k}(x-a)^{k+1}}{(k+1) !} \alpha\right) \text { never is }-\infty \text { for any component, } \\
{\left[F-\frac{r c^{k}(x-a)^{k+1}}{(k+1) !} \alpha\right]_{x=a}=F(a) \geqq \theta .}
\end{gathered}
$$

It is known $[1, \S 11.83$, Lemma 2] that these conditions yield $F$ $\geqq\left(r c^{k}(x-a)^{k+1} /(k+1) !\right) \alpha$. Then using (2) and (3) we have

$$
D^{+} F \geqq M F \geqq M \frac{r c^{k}(x-a)^{k+1}}{(k+1) !} \alpha \geqq \frac{r c^{k+1}(x-a)^{k+1}}{(k+1) !} \alpha
$$

almost everywhere.

Letting $n$ go to infinity, we have $D^{+} F \geqq \theta$ almost everywhere. As $F(a) \geqq \theta$ and $D^{+} F$ never is $-\infty$ for any component, by the result from [1] used above, we have $F \geqq \theta$ on $[a, b]$.

Lemma 2. On the interval $[a, b]$ let:

(1) $F$ be continuous, $F \leqq \theta, D^{+} F$ never be $-\infty$ for any component, and $F(a)=\theta$;

(2) $M$ be essentially bounded above;

(3) $D^{+} F \geqq M F$ almost everywhere.

Then $F=\theta$ on $[a, b]$.

Proof. By $F \leqq \theta$ and (3) we have $D^{+} F \geqq M F=\left(M_{1}+M_{2}\right) F=M_{1} F+M_{2} F \geqq M_{1} F$ almost everywhere By Lemma $1, F \geqq \theta$ on $[a, b]$; so $F=\theta$ on $[a, b]$.

Theorem 1. On the interval $[a, b]$ let: 
(1) $F_{2}$ be continuous, $D^{+} F_{2}$ never be $-\infty$ for any component, and $F(a) \geqq \theta$;

(2) $M$ be non-negative almost everywhere off the main diagonal and $M$ be essentially bounded above;

(3) $D^{+} F \geqq M F$ almost everywhere.

Then $F \geqq \theta$ on $[a, b]$.

Proof. We shall show first that $D^{+} F_{2} \geqq M F_{2}$ almost everywhere. We have two cases for the components of $F$.

Case I. $f_{i} \leqq 0$ on $(x, x+h]$. For this situation $f_{i 2}=f_{i}$ on $(x, x+h]$. Thus $D^{+} f_{i 2}(x)$ is not less than $D^{+} f_{i}(x)$. Note that if $D^{+} F \geqq M F$ at $x$; $D^{+} f_{i}$ cannot be $-\infty$ at $x, f_{i}(x)$ must be nonpositive, and $f_{i}(x)=f_{i 2}(x)$. Then for almost all such values of $x$

$$
\begin{aligned}
D^{+} f_{i 2}(x) & \geqq m_{i i}(x) f_{i}(x)+\sum_{j \neq i} m_{i j}(x) f_{j}(x) \\
& \geqq m_{i i}(x) f_{i 2}(x)+\sum_{j \neq i} m_{i j}(x) f_{j 2}(x) \\
& =\sum_{j=1}^{n} m_{i j}(x) f_{j 2}(x) .
\end{aligned}
$$

Case II. For every positive $h, f_{i}$ is somewhere positive on $(x, x+h]$. Then $f_{i 2}$ is 0 somewhere inside every such interval; so $f_{i 2}(x)=0$ by continuity and $D^{+} f_{i 2}(x)=0$. Thus for almost all such values of $x$

$$
\begin{aligned}
D^{+} f_{i 2}(x)=0=m_{i i}(x) f_{i 2}(x) & \geqq m_{i i}(x) f_{i 2}(x)+\sum_{i \neq j} m_{i j}(x) f_{j 2}(x) \\
& =\sum_{j=1}^{n} m_{i j}(x) f_{j 2}(x) .
\end{aligned}
$$

Since $F(a) \geqq \theta$ implies $F_{2}(a)=\theta$, by Lemma $2, F_{2}=\theta$ on $[a, b]$. Thus $F \geqq \theta$ on $[a, b]$.

Corollary 1.1. Change (1) of Theorem 1 to

$\left(1^{\prime}\right) F$ be continuous, $D^{+} F$ never be $-\infty$ for any component, $F(a) \geqq \theta$. Then $F \geqq \theta$ on $[a, b]$.

Proof. $F_{2}$ is continuous on $[a, b]$. Noting Cases I and II of Theorem 1 , we see that

$$
D^{+} f_{i 2} \geqq D^{+} f_{i} \quad \text { or } \quad D^{+} f_{i 2}=0 .
$$

Thus $D^{+} F_{2}$ never has $-\infty$ as a component. Then Theorem 1 yields the desired conclusion.

TheOREM 2. On the interval $[a, b]$ let: 
(1) $G$ be a solution almost everywhere of $D^{+} Y=M Y+K$ such that $G(a) \leqq F(a)$;

(2) $(F-G)_{2}$ be continuous and $D^{+}(F-G)_{2}$ never be $-\infty$ for any component;

(3) $M$ be non-negative almost everywhere off the main diagonal and $M$ be essentially bounded above;

(4) $D^{+} F \geqq M F+K$ almost everywhere.

Then $F \geqq G$ on $[a, b]$.

Proof. Let $P=F-G$. Then since $D^{+} G$ is finite almost everywhere, $D^{+} F-D^{+} G$ is defined almost everywhere; and

$$
D^{+} P \geqq D^{+} F-D^{+} G \geqq M F+K-M G-K=M(F-G)=M P
$$

almost everywhere.

Also $P_{2}(a)=(F-G)_{2}(a)=\theta$, since $G(a) \leqq F(a)$.

The other conditions required to apply Theorem 1 to the function $P$ are explicitly hypotheses of Theorem 2 . Therefore $P \geqq \theta$ and $F \geqq G$ on $[a, b]$.

The result (Theorem 3 below) which is related to Corollary 1.1 as Theorem 2 is related to Theorem 1 is perhaps more natural to use since the hypotheses need not be stated in terms of $F-G$.

Theorem 3. On the interval $[a, b]$ let:

(1) $G$ be a continuous solution of $D^{+} Y=M Y+K$ such that $G(a)$ $\leqq F(a)$;

(2) $F$ be continuous, $D^{+} F$ never be $-\infty$ for any component;

(3) $M$ be non-negative almost everywhere off the main diagonal and $M$ be essentially bounded above;

(4) $D^{+} F \geqq M F+K$ almost everywhere.

Then $F \geqq G$ on $[a, b]$.

Proof. Let $P=F-G$. Then $P$ is continuous. Also

$$
P(a)=F(a)-G(a) \geqq \theta .
$$

Since $D^{+} G$ is always finite, and $D^{+} F$ is never $-\infty$ for any component, $D^{+} P$ is never $-\infty$ for any component. Finally, almost everywhere

$$
D^{+} P \geqq D^{+} F-D^{+} G \geqq M P .
$$

Using hypothesis (3) and Corollary 1.1 , we have that $P \geqq \theta$ or $F \geqq G$ on $[a, b]$.

3. Examples. In this section we consider two examples which show that neither the boundedness condition on $M_{1}$ nor the non-negativity 
condition on $M$ can be dispensed with completely.

Let the functions $f$ and $m$ be defined by

$$
f(x)=-x^{2} \text { and }\left\{\begin{array}{l}
m(x)=2 / x \quad(x>0), \\
m(0)=0 .
\end{array}\right.
$$

Then $f^{\prime}(0)=0=0 \cdot 0=m(0) f(0)$; and for $x>0, f^{\prime}=-2 x=m f$.

All the hypotheses of Theorem 1 are satisfied except that $m$ is not essentially bounded above. Also the conclusion of Theorem 1 is seen not to hold.

Suppose next that the matrix $M$ is $3 \times 3$ and constant, and that the term $m_{21}$ is negative. Let $f_{1}=\left(1-2 / m_{21}\right) x, f_{2}=-x^{2}$, and $f_{3}$ $=\left[m_{31}\left(1-2 / m_{21}\right) / 2+1\right] x^{2}$. It is easily verified that in a suitably small right neighborhood of $x=0$ all the hypotheses of Theorem 1 are satisfied except that $M$ is not non-negative almost everywhere off the main diagonal. The conclusion of Theorem 1 is seen not to hold.

The latter example is readily generalized to the $n$-dimensional case. Also by letting $F$ be $\theta$ on the appropriate initial portion of the interval $[a, b]$ and replacing $x$ by $x-c$ where $c$ is appropriate, the example can be generalized to any interval $[a, b]$.

The latter example shows that any conditions on the elements of $M$ which will insure the conclusion of Theorem 1 for all $F$ must insure the conditions we have imposed as regards the case that $M$ is constant. It would be of interest to have examples which violate the conclusion of Theorem 1 whenever $m_{21}$ is negative on a set of positive measure. A similar remark applies to the first example.

4. A more general inequality. In this section we consider inequalities of the form $D^{+} F \geqq G(F, x)$. In certain cases these can be reduced to inequalities of the type previously discussed. The conditions imposed on $G$ are obviously chosen just so as to permit this reduction.

THEOREM 4. Let $U$ be an open neighborhood of $(F(a), a)$ in $(n+1)$ dimensions. On the interval $[a, b]$, let:

(1) $F$ be continuous and $(F, x)$ remain in $U$;

(2) For almost all $x, g_{i}(Y, x)$ be nondecreasing in $y_{j}$ for $j \neq i$ when $(Y, x)$ is in $U$;

(3) $g_{i}(Y, x)$ satisfy an upper Lipschitz condition on $U$ in $y_{j} u n i$ formly with respect to the $y_{k}$ 's and almost all $x$;

(4) $D^{+} F \geqq G(F, x)$.

Then there is a matrix $M$, non-negative almost everywhere off the main diagonal and essentially bounded above, such that $D^{+} F \geqq M F$ $+G(F(a), x)$. 
Proof. We have

$$
\begin{aligned}
g_{i}(F, x)= & \sum_{j=1}^{n}\left[g_{i}\left(f_{1}(a), f_{2}(a), \cdots, f_{j-1}(a), f_{j}, f_{j+1}, \cdots, f_{n}, x\right)\right. \\
& \left.-g_{i}\left(f_{1}(a), f_{2}(a), \cdots, f_{j-1}(a), f_{j}(a), f_{j+1}, \cdots, f_{n}, x\right)\right] \\
& +g_{i}(F(a), x) .
\end{aligned}
$$

For $f_{j} \neq f_{j}(a)$, let

$$
\begin{array}{r}
m_{i j}(x)=\frac{g_{i}\left(f_{1}(a), f_{2}(a), \cdots, f_{j-1}(a), f_{j}, f_{j+1}, \cdots, f_{n}, x\right)}{f_{j}-f_{j}(a)} \\
-\frac{g_{i}\left(f_{1}(a), f_{2}(a), \cdots, f_{j-1}(a), f_{j}(a), f_{j+1}, \cdots, f_{n}, x\right)}{f_{j}-f_{j}(a)} .
\end{array}
$$

For $f_{j}=f_{j}(a)$, let $m_{i j}(x)=0$. Then by (3), the $m_{i j}(x)$ are essentially bounded above. Moreover, by (2), when $i \neq j$ we have $m_{i j}(x) \geqq 0$ for almost all values of $x$.

Thus we can write

$$
D^{+} F \geqq G(F, x)=M F+G(F(a), x)
$$

where $M$ has the desired properties.

\section{REFERENCE}

1. E. C. Titchmarsh, The theory of functions, 2d ed., Oxford University Press, 1950 SANDia CoRPoration 